\title{
Atomic Resolution Imaging and Quantitative Elemental Mapping of the Misfit Dislocation Core Phase in Multicomponent Oxides
}

\author{
N. Bagués ${ }^{1}$, J. Santiso ${ }^{2}$, B. D. Esser ${ }^{1}$, R. E. A. Williams ${ }^{1}$, D. W. McComb ${ }^{1}$, Z. Konstantinovic ${ }^{3}$, Ll. \\ Balcells $^{4}$ and F. Sandiumenge ${ }^{4}$ \\ 1. Center for Electron Microscopy and Analysis, The Ohio State University, Columbus, OH. \\ 2. Catalan Institute of Nanoscience and Nanotechnology (ICN2), CSIC and BIST, Campus UAB, \\ Bellaterra, Spain. \\ 3. Center for Solid State Physics and New Materials, Institute of Physics Belgrade, University of \\ Belgrade, Serbia. \\ 4. Institut de Ciència de Materials de Barcelona, ICMAB-CSIC, Campus de la UAB, Bellaterra, Spain.
}

The relaxation mechanisms of lattice mismatched heteroepitaxial films may accommodate defects, such as misfit dislocations (MDs). Such MDs are located at the interface between two different compounds and can be considered linear defects exhibiting their own distinct structural, chemical, and physical properties. Recent studies have highlighted the strong potential of buried and laterally ordered MD networks in creating a variety of periodic strain-induced functional patterns in oxide thin films [1-3]. These recent findings have motivated analysis of the atomic and chemical structure of the MD phase in complex oxides, in view of the development of functional dislocation-based nanostructures.

In this work, we combine imaging and spectroscopic techniques to determine the complex structure of MDs in the perovskite type $\mathrm{La}_{0.67} \mathrm{Sr}_{0.33} \mathrm{MnO}_{3} / \mathrm{LaAlO}_{3}$ (LSMO/LAO) heteroepitaxial system. The atomic structure of the core and surrounding area of the MDs is analyzed by high resolution (HR) transmission electron microscopy (TEM) and aberration-corrected scanning (S)TEM, as well as its chemical and electronic configuration by means of energy dispersive x-ray (EDS) spectral mapping and electron energy-loss (EELS) spectrum images.

A high angle annular dark field (HAADF) STEM image of a MD core at the LSMO-LAO interface is shown in Figure 1. This MD is characterized by a $a[010]$ Burgers vector parallel to the LSMO-LAO interface, where $a$ is the LAO lattice parameter. A careful analysis of the HR-TEM and HR-HAADF data reveals that the dislocation core is dissociated and presents two extra half-planes separated by one unit cell. The strain field associated with this dislocation is characterized by tensile and compressive regions extending above and below the dislocation glide plane, which is parallel to LSMO-LAO interface. The HAADF images of the MD core indicate the formation of a $\mathrm{La}_{\mathrm{Mn}}^{\mathrm{x}}$ anti-site defect in the tensile zone to accommodate the tensile strain. The atomic resolution EDS and EELS analyses reveal that, while the position of the LSMO-LAO interface is blurred by cation intermixing, oxygen vacancies selectively accumulate in the tensile region of the dislocation strain field. Such accumulation of vacancies is accompanied by the reduction of $\mathrm{Mn}$ cations in the same area. The imbalance between the concentration of oxygen vacancies and the rate of Sr diffusion out of the core results in a positive net charge $q \approx+0.3 \pm 0.1$ localized in the tensile region of the dislocation, while the compressive region remains neutral. The basic mechanisms involve in the distribution of cations and oxygen vacancies around the MD core are schematically summarized in the right panel of Figure 1. These results highlight a prototypical core model for perovskite-based heteroepitaxial systems and offer insights into predictive manipulation of MD properties [4, 5]. 
References:

[1] C.-P. Chang et al, Nature Communications 5 (2014), p. 3522.

[2] S. Pandya et al, Scientific Reports 6 (2016), p. 26075.

[3] F. Sandiumenge et al, Advanced Materials Interfaces 3 (2016), p. 1600106.

[4] N. Bagués et al, Advanced Functional Materials (2017), p. 1704437.

[5] N.B. thanks the Spanish MINECO for the financial support through the FPI program. This research was funded by Spanish MINECO (Grant Numbers: SEV-2015-0496, MAT2015-71664-R, SEV-20130295), European Union Horizon 2020 research and innovation programme (Grant Number: 645658), Generalitat de Catalunya (Grant Numbers: 2014 SGR 501, 2014 SGR 1216). B.D.E. and D.W.M. acknowledge support from the Center for Emergent Materials at the Ohio State University, a National Science Foundation Materials Research Science and Engineering Center (Grant Number: DMR1420451).
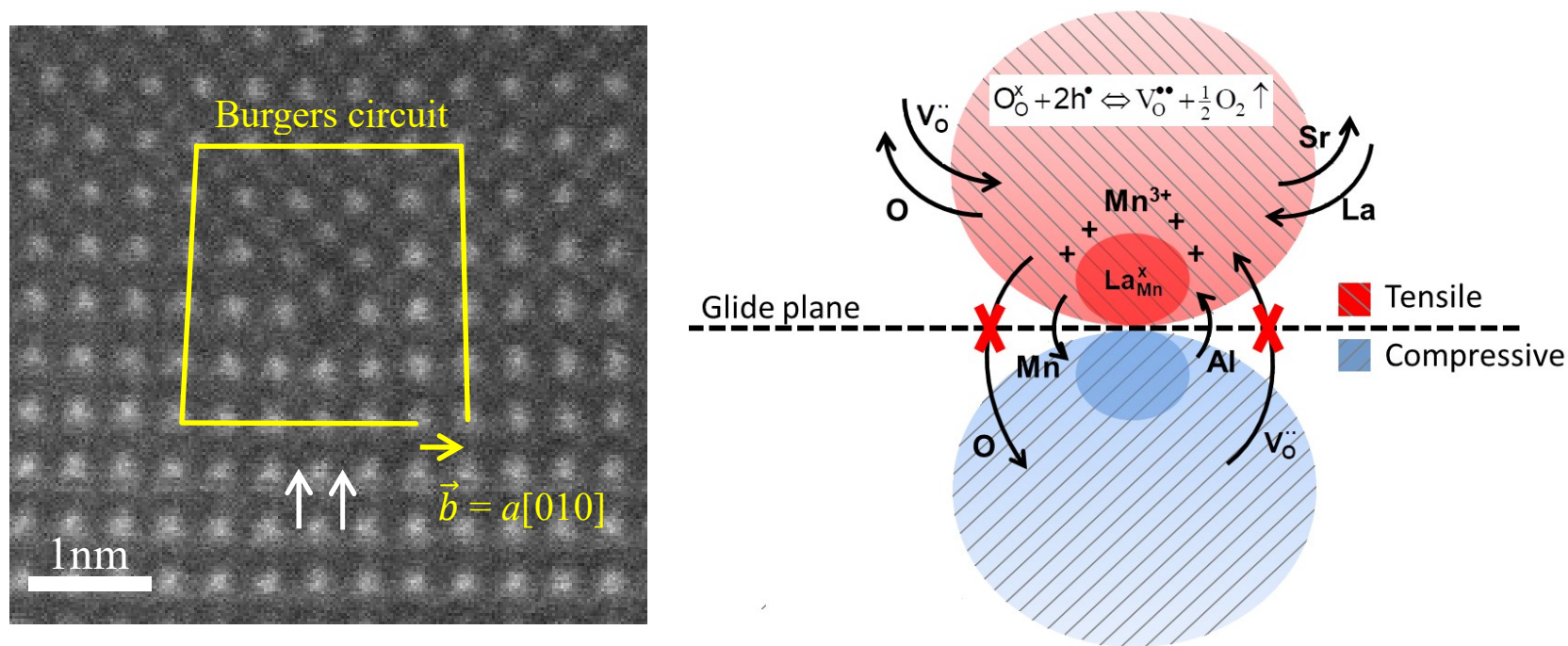

Figure 1. Left panel, an atomic resolution HAADF image, viewed along the [100] zone axis, of a MD. Lines draw a Burgers circuit yielding the Burgers vector $\vec{b}=a[010]$ (yellow arrow). The two white arrows indicate the position of the two extra half-planes of the dissociated core. Right panel, schematic illustration of the basic mechanisms operating in the MD core. Red and blue represent tensile and compressive regions above and below the dislocation glide plane, respectively. The redox reaction indicated in the tensile region is displaced to the right, favoring the formation of electron donor oxygen vacancies, $\mathrm{V}_{\mathrm{O}}$. Each vacancy nominally releases two electrons which can reduce two neighboring $\mathrm{Mn}^{4+}$ cations. The imbalance between the rate of Sr diffusion out of the core region and the concentration of oxygen vacancies results in a positive charge $(+)$ in the tensile region. The glide plane acts as a barrier for the redistribution of vacancies as indicated by crossed-out pathways. $\mathrm{La}_{\mathrm{Mn}}^{\mathrm{x}}$ is the anti-site defect formed to accommodate the tensile strain. Adapted from reference [4]. 